\title{
Confirmação da sinonímia de Gynaecotyla jaegerskioeldi (Travassos) (Digenea, Microphallidae) com Gynaecotyla adunca (Linton) (Digenea, Gynaecotylinae)
}

\author{
Luís C. Muniz-Pereira ${ }^{1,2}$, Vanessa Santos de Arruda ${ }^{1} \&$ Roberto Magalhães Pinto ${ }^{1,3}$ \\ ${ }^{1}$ Laboratório de Helmintos Parasitos de Vertebrados, Departamento de Helmintologia, Instituto Oswaldo Cruz. Avenida Brasil \\ 4365, 21040-900 Rio de Janeiro, Rio de Janeiro, Brasil. \\ 2 Autor correspondente. E-mail: Imuniz@ioc.fiocruz.br \\ ${ }^{3}$ Bolsista do CNPq.
}

\begin{abstract}
Confirmation of the synonymy of Gynaecotyla jaegerskioeldi (Travassos) (Digenea, Microphallidae) with Gynaecotyla adunca (Linton) (Digenea, Gynaecotylinae). The synonymy of Gynaecotyla jaegerskioeldi (Travassos, 1920) Yamaguti, 1939 with Gynaecotyla adunca (Linton, 1905) Yamaguti, 1939 is confirmed, on the basis of the detailed morphological study of the cornucotyle of specimens of both referred species. The cornucotyle is a hermaphroditic organ that can be formed by the ovijector and the ejaculatory duct and is an important specific diagnostic character.

KEY WORDS. Birds, Brazil, cornucotyle, mammals, morphology, trematodes.
\end{abstract}

RESUMO. A sinonímia de Gynaecotyla jaegerskioeldi (Travassos, 1920) Yamaguti, 1939 com Gynaecotyla adunca (Linton, 1905) Yamaguti, 1939 é confirmada, com base no estudo morfológico detalhado do cornucótile de espécimes das duas espécies. $O$ cornucótile é um órgão hermafrodita que pode ser formado pelo ovejetor e o duto ejaculador e é um caráter importante na diferenciação das espécies do gênero.

PALAVRAS CHAVE. Aves, Brasil, cornucótile, mamíferos, morfologia, trematódeos.

Linton (1905) descreveu Distomum aduncum coletada em intestino de Opsanus tau (Linnaeus, 1766) na Carolina do Norte. Em 1928 o mesmo autor transfere esta espécie para Levinseniella.

Travassos (1920) descreveu sob o nome Levinseniella jaegerskioeldi espécimes provenientes de Didelphis aurita WiedNeuwied, 1826 e Rallus cayenensis Linnaeus, 1758, coletados em Manguinhos, Rio de Janeiro, Rio de Janeiro.

YAmaguti (1939) propôs Gynaecotyla, com a espécie tipo G. squatarolae (Yamaguti, 1934) e incluiu neste G. adunca e $G$. jaegerskioeldi entre outras espécies, baseado na suposição de que nestas o cirro é modificado em um órgão copulador masculino, o poro genital feminino é provido de uma ventosa que se abre separadamente do poro masculino e existe uma bolsa do cirro, diferente de Levinseniella Stiles and Hassal, 1901 que possuia uma papila simples, quatro digitações no átrio genital e um pequeno cirro.

Rankin (1939) descreveu Cornucopula, com a espécie tipo C. jaegerskioeldi e acrescenta neste as mesmas espécies de Gynaecotyla de YAMAGUTI (1939), considerando que estas possuem um segundo acetábulo que é descrito erroneamente como ventosa genital, uma bolsa do cirro e um complexo órgão copulador masculino ao invés de papilas acessórias que são características de Levinseniella.
Ao descrever o ciclo biológico de G. nassicola (Cable \& Hunninen, 1938), RANKIN (1940) reconheceu a sinonimia entre Cornucopula e Gynaecotyla e adota este segundo nome de acordo com a lei da prioridade.

Hopkins (1940), talvez desconhecendo o trabalho de YAmaguti (1939), descreveu o sistema excretor de Cornucopula adunca. Hunter (1952) descreveu esta espécie através de espécimes coletados em Rynchops nigra Linnaeus, 1758, Sterna hirundo Linnaeus, 1758 e $S$. albifrons Pallas, 1764.

Deblock \& Pearson (1968) ao discutirem a taxonomia dos Gynaecotylinae Guschanskaia, 1952 e proporem uma chave de identificação de espécies, consideram G. jaegerskioeldi sinônima de G. adunca ou species inquirenda, enquanto YAMAGUTI (1971) mantém $G$. adunca e G. jaegerskioeldi como espécies distintas.

Ao complementar a descrição de G. jaegerskioeldi, Dевцоск (1972) considerou esta sinônima de G. adunca, com base na comparação entre os cornucótiles, embora esta estrutura não tivesse sido descrita detalhadamente em ambas as espécies.

Ao descrever o cornucótile de várias espécies, entre elas de G. adunca pela primeira vez, Dевцоск (1974) preconizou toda nomenclatura referente a esta estrutura e ao apresentar uma chave de identificação de espécies, o autor considera $G$. jaegerskioeldi sinônimo "provável" de G. adunca.

Revista Brasileira de Zoologia 21 (4): 801-804, dezembro 2004 
A dúvida com relação à sinonimia entre G. adunca e $G$. jaegerskioeldi então permanecia, visto que o cornucótile de espécimes até então identificados como G. jaegerskioeldi nunca havia sido descrito, o que é feito no presente trabalho, permitindo a confirmação da sinonímia entre as duas espécies, prevalecendo a mais antiga, G. adunca.

O cornucótile, que é um órgão hermafrodita por ser constituído pelo ovejetor e pelo canal ejaculador, é de grande importância na diferenciação das espécies segundo DeвLock (1974).

Novos registros de hospedeiros para G. adunca (= $G$ jaegerskioeldi) foram feitos por Vicente et al. (1983) e ARRUDA et al. (2001).

\section{MATERIAL E MÉTODOS}

Foram estudados 27 espécimes coletados em intestino de Aramides cajanea (Mueller, 1776), Aramides mangle (Spix, 1825), Butorides striatus (Linnaeus, 1758), Florida caerulea (Linnaeus, 1758), Nyctanassa violacea (Linnaeus, 1758), Pitangus sulphuratus (Linnaeus, 1766) e Rallus longirostris (Boddaert, 1783), entre 1918 e 1949 em Manguinhos, Rio de Janeiro, RJ, Brasil e depositados na Coleção Helmintológica do Instituto Oswaldo Cruz (CHIOC).

As amostras em meio líquido estão conservadas em formol acético e as amostras montadas definitivamente estão preservadas em bálsamo do Canadá.

No processamento das amostras em meio líquido, foram feitas a coloração por carmim, através do método regressivo com posterior desidratação e diafanização em creosoto. Para a montagem definitiva dos helmintos utilizou-se bálsamo do Canadá.

A nomenclatura utilizada na descrição do cornucótile está de acordo com Deblock (1974) e as medidas estão em micrometros.

O exame do material foi realizado em microscópio Olympus e a fotomicrografia foi obtida em microscópio modelo Zeiss com sistema Axiophot com a opção do recurso CID (Contraste de Interferência Diferencial). A imagem foi processada através do sistema KS300, sendo posteriormente editada com o auxílio do programa Adobe Photoshop ${ }^{\circledR}$.

Os desenhos foram feitos através de um microscópio Olympus ${ }^{\circledR}$ CBA com um tubo de desenho conectado. O material montado definitivamente foi depositado na CHIOC.

\section{RESULTADOS}

\section{Gynaecotyla adunca (Linton, 1905) Yamaguti, 1939 Figs $1-4$}

Distomum aduncum Linton, 1905. Gynaecotyla jaegerskioeldi Travassos, 1920. Levinseniella jaegerskioeldi; Travassos, 1920.

Descrição dos espécimes estudados (medidas baseadas em 27 espécimes): corpo piriforme a alongado, com 414-840 (563,9) de comprimento, 331-448 (391) de largura máxima na altura dos testículos. A razão entre comprimento/largura é de 1,0-2,0 $(1,4)$. Tegumento com espinhos diminutos até a região testicular. Ventosa oral (V.O.) subterminal, medindo 43-72 $(53,7)$ de comprimento, 54-72 $(62,8)$ de largura. Pré-faringe com 1-57 $(16,9)$ de comprimento. Faringe muscular com 21-54 $(36,8)$ de comprimento, 28-50 $(37,1)$ de largura. Esôfago medindo 32-122 $(79,3)$ de comprimento. Cecos intestinais com paredes espessas, terminando na zona testicular. Presença de duas ventosas ventrais, pós-equatoriais, pós-cecais, medianas e paralelas. Ventosa ventral poral (V.V.P.) menor, situada no campo mediano ao lado do átrio genital com 39-57 $(47,6)$ de comprimento, 36-63 $(43,9)$ de largura. Ventosa ventral antiporal (V.V.A.), sempre maior que a poral, ligeiramente a esquerda do corpo, ao lado do ovário, medindo 46-68 $(54,8)$ de comprimento, 43-57 $(51,1)$ de largura. Aparelho copulador - cornucótile - (Cct.) muscular, encerrado no átrio genital situado destro lateralmente a V.V.P, com 61-93 $(78,5)$ de comprimento, 75-108 $(96,1)$ de largura. Bolsa vesículoprostática (B.V.P.) transversal, pós-bifurcal e pré-acetabular, medindo 162-198 $(169,5)$ de comprimento, encerrando uma vesícula seminal. Vesícula seminal sacular medindo 90-162 (119) de comprimento. Presença de três feixes musculares sob o arco inferior da B.V.P. Ovário (Ov.) esférico, pós-equatorial, pré-testicular, pós-bifurcal, na mesma zona acetabular, no lado esquerdo do corpo, medindo 46-100 $(74,4)$ de comprimento, 68-108 (89) de largura. Testículos ovais a esféricos, pós-equatorial, na mesma zona e com campos afastados; o direito medindo 39-75 $(55,7)$ de comprimento, $39-118(88,4)$ de largura e o esquerdo com 36-93 $(58,6)$ de comprimento, 39-111 $(85,8)$ de largura. Vasos deferentes passam entre o ovário e a V.V.A. entrando na B.V.P. e formando a vesícula seminal. Útero pós-ovariano com alças amplas, ocupando a metade posterior do corpo, sobrepondo-se aos testículos e a vitelária. Metratermo musculoso, bem individualizado situado entre a V.V.A. e o Cct., dando continuação a um ovejetor. Vitelária lateral, discretamente assimétrica, formando dois campos que alcançam a região testicular e situada na metade posterior do corpo. Ductos vitelínicos transversais, pós-acetabulares e ventrais aos testículos. Numerosos ovos operculados com 16-18 $(17,2)$ de comprimento, 10 de largura. Cct. $>$ V.O. $\geq$ V.V.A. $>$ V.V.P.

Descrição do cornucótile: composto por um crescente externo maior, musculoso e ventral, desprovido de verrucosidades e portando duas peças esclerotinizadas - uma mediana e uma distal; um crescente interno menor, musculoso e dorsal, portando uma peça esclerotinizada e parte da superfície com verrucosidades cônicas; placas que são pontos de inserção de dois feixes musculares transversais, no qual o mais largo parte medianamente e o mais estreito distalmente para o arco inferior da bolsa B.V.P.; um terceiro feixe muscular longitudinal ventral que liga a porção proximal da borda anterior da B.V.P. à porção esquerda anterior do átrio genital; um ducto ejaculador saindo ventralmente da B.V.P. e entrando no cornucótile; um ovejetor passando ventralmente ao ducto ejaculador partindo

Revista Brasileira de Zoologia 21 (4): 801-804, dezembro 2004 

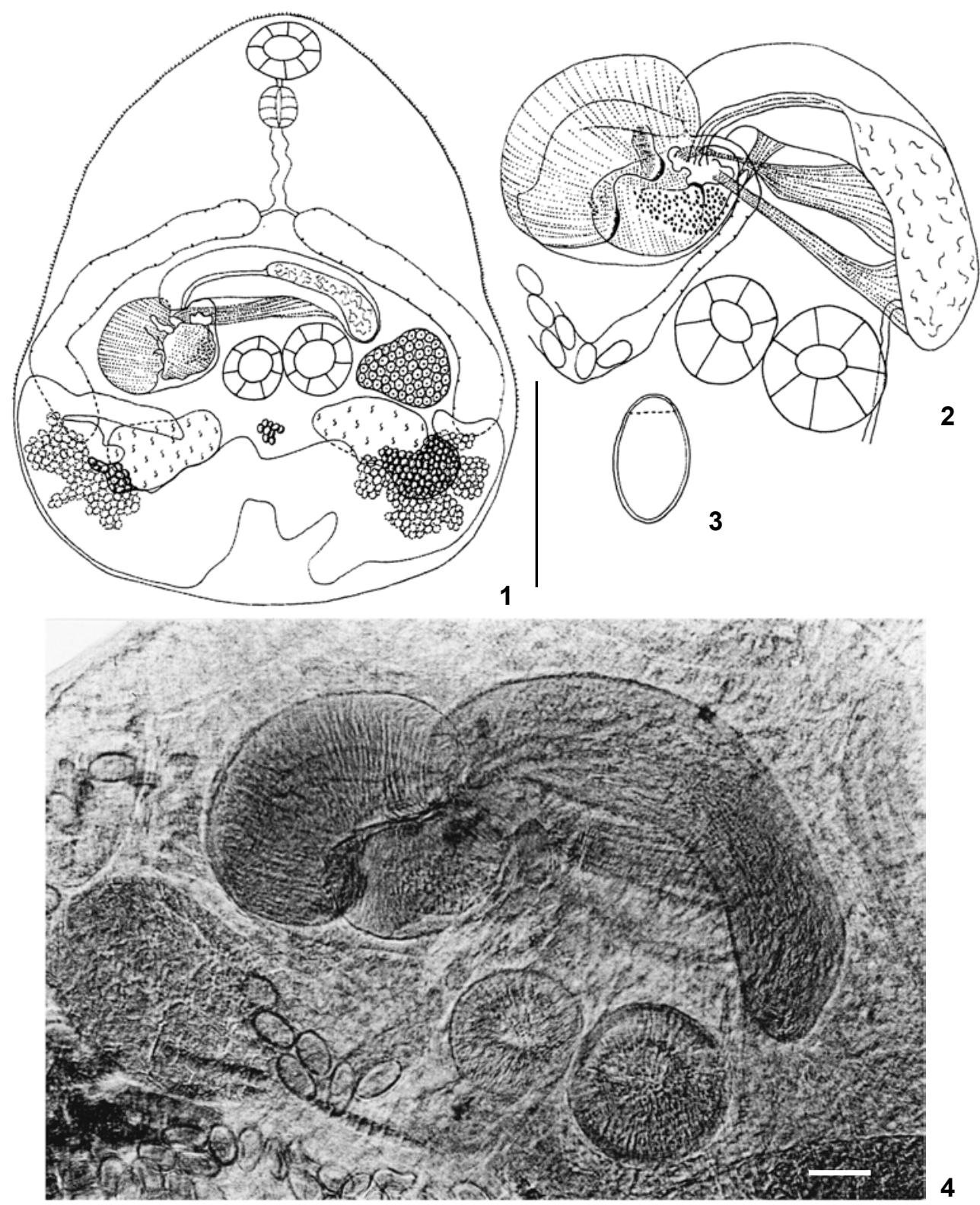

Figuras 1-4. Gynaecotyla adunca: (1) adulto, vista dorsal, CHIOC $n^{\circ}$. 2237.; (2) detalhe do cornucótile, vista dorsal, CHIOC $\mathrm{n}^{\circ}$. 2236; (3) ovo, CHIOC $n^{\circ}$. 2236; (4) detalhe do cornucótile e das duas ventosas ventrais, obtido pelo sistema de contraste de interferência diferencial (CID), $\mathrm{CHIOC} \mathrm{n}^{\circ}$. 34582. Escalas $=0,02 \mathrm{~mm}$.

de um metratermo ventral aos dois feixes musculares transversais e dorsal ao feixe muscular longitudinal; átrio genital sinistral e abrindo-se anteriormente, próximo aos feixes musculares.

Hospedeiros: Butorides striatus (Linnaeus ,1758), Florida caerulea (Linnaeus, 1758), Nyctanassa violacea (Linnaeus, 1758), Aramides cajanea (Mueller, 1776), Pitangus sulphuratus (Linnaeus, 1766), Rallus longirostris (Boddaert, 1783) e Aramides mangle (Spix, 1825).
Proveniência: Manguinhos, Rio de Janeiro, Rio de Janeiro. Localização: intestino.

Espécimes estudados: 2069 (holótipo), 2231 (6 espécimes), 2236, 2237 e 2645 (2 espécimes), 2780, 12092a ( 2 espécimes), 12094 (9 espécimes), 25554, 34582 a-d (4 espécimes).

Outros hospedeiros: Didelphis aurita Wied-Neuwied, 1826, Didelphis marsupialis Linnaeus, 1758 segundo YAMAGUTI (1971) e guaxinim segundo Harkema \& Miller (1961). 


\section{DISCUSSÃO}

Os cornucótiles de G. jaegerskioeldi e G. adunca são extremamente semelhantes, sendo a única diferença entre eles a presença de um terceiro feixe muscular longitudinal em $G$. jaegerskioeldi. Porém este feixe pode não ter sido visualizado por Dевцоск (1974) em G. adunca, visto que esta estrutura nem sempre era visível em todos os espécimes de G. jaegerskioeldi, talvez devido a alguma falha de processamento. A variação na posição dos espécimes na lâmina também pode dificultar a visualização das estruturas do cornucótile. No entanto, não há diferenças significativas entre as peças que formam os cornucótiles e demais caracteres do corpo de ambas espécies que justifiquem a separação destas.

Examinando os trabalhos de RANKIN $(1939,1940)$, DeвLock \& Pearson (1968), Yamaguti (1971) e Deblock (1974), verifica-se que as demais espécies do gênero, quando comparadas com $G$. adunca, podem ser diferenciadas em vários aspectos: $G$. squatarola possui ventosa oral menor que a ventosa ventral antiporal, ventosa oral igual à ventosa ventral poral, cornucótile igual à ventosa ventral antiporal, cornucótile com ausência de verrucosidades e cecos curtos não alcançando a região testicular. Gynaecotyla bridgmani Deblock, 1974 (= G. squatarole) segundo Dевцоск (1974) possui a relação ventosa ventral poral/ ventosa ventral antiporal maior, cornucótile aproximadamente do mesmo tamanho que a ventosa ventral poral e desprovido de peças esclerotinizadas, testículos com campos juntos e tocando a ventosa ventral poral e vitelária em folículos grandes e simétricos. Gynaecotyla sp. nº 1 Deblock, 1974 possui cornucótile menor que a ventosa ventral antiporal e a ventosa ventral poral do mesmo tamanho do cornucótile. Gynaecotyla brisbanensis Deblock \& Pearson, 1968 possui a relação ventosa ventral poral/ventosa ventral antiporal maior, cornucótile aproximadamente do mesmo tamanho da ventosa ventral poral, pré-faringe mais longa e cornucótile com uma lâmina quitinosa entre os dois crescentes e desprovido de feixes musculares. Gynaecotyla nassicola Cable \& Hunninen, 1938 (= G. sippiwissettensis Rankin, 1939) segundo Dевцоск (1974) possui tamanho menor, ventosa ventral poral do mesmo tamanho da ventosa ventral antiporal e vitelária constituída por dois grupos simétricos de grandes folículos. Gynaecotyla longiintestinata Leonov, 1958 (= G. gallica Rebecq, 1961) segundo Dевцоск (1974) possui ovos maiores e cornucótile menor com crescentes superpostos. Gynaecotyla beihaiensis Ke, 1980 possui ventosa ventral antiporal do mesmo tamanho que a ventosa ventral poral e cornucótile bem pequeno $(27 \mu \mathrm{m})$.

\section{REFERÊNCIAS BIBLIOGRÁFICAS}

Arruda, V.S.; R.M. Pinto \& L.C. Muniz-Pereira. 2001. New host and geographical records for helminths parasites of Ardeidae (Aves: Ciconiiformes) in Brazil. Revista Brasileira de Zoologia, Curitiba, 18: 225-232.

Deвlock, S. 1972. Contribution à l'étude des Microphallidae
Travassos, 1920 (Trematoda) XXIII. Les espèces décrites au Brésil par L. Travassos. Annales de Parasitologie Humaine et Comparée, Paris, 47: 77-89.

. 1974. Contribution à l'étude des Microphallidae Travassos, 1920 (Trematoda) XXIX. A propos d'espèces décrites au Japon par S. Yamaguti B. Le genre Gynaecotyla Yamaguti, 1939. Annales de Parasitologie Humaine et Comparée, Paris, 49: 319-335.

Deblock, S.; J.C. Pearson. 1968. Contribution à l'étude des Microphallidae Travassos, 1920 (Trematoda). XVI. Trois Gynaecotylinae nouveaux d'Australie. Considérations systématiques. Annales de Parasitologie Humaine et Comparée, Paris, 43: 131-148.

Harkema, R. \& G.C. Miller. 1961. Observations on parasitism of the raccoon of Cape Island, S. C. Journal of Parasitology, Lawrence, 47: 41.

Hopkins, S.H. 1940. The excretory systems of Tergestia Stossich 1899 and Cornucopula adunca (Linton 1905) (Trematoda). Transactions of the American Micoscopical Society, Menasha, 59: 281-284.

HunTER, W.S. 1952. Contributions to the morphology and lifehistory of Gynaecotyla adunca (Linton, 1905) (Trematoda: Microphallidae). Journal of Parasitology, Lawrence, 38: 308-314.

LinTon, E. 1905. Parasites of fishes of Beaufort, North Carolina. Bulletin of the United States Bureau of Fisheries, Washington, 24: 409.

RANKIN JR., J.S. 1939. Studies on the trematode family Microphallidae Travassos, 1921 I. The genus Levinseniella Stiles and Hassal, 1901 and description of a new genus Cornucopula. Transactions of the American Microscopical Society, Menasha, 58: 431-447.

- 1940. Studies on the trematode family Microphallidae Travassos, 1921 IV. The life cycle and ecology of Gynaecotyla nassicola (Cable and Hunninen, 1938) Yamaguti, 1939. Biological Bulletin, Stanford, 3: 439-451.

TRavassos, L. 1920. Contribuição para conhecimento da fauna helmintológica brasileira - IX. Sôbre as espécies da subfamília Microphalinae Ward, 1901. Archivos da Escola Superior de Agricultura e Medicina Veterinária, Niterói, 4: 85-91.

Vicente, J.J.; R.M. Pinto \& D. Noronha. 1983. Skrjabinoclava tupacincai Freitas, Vicente \& Ibañez, 1970 in Brazil and some other helminths from Tyrannidae birds. Memórias do Instituto Oswaldo Cruz, Rio de Janeiro, 78: 95-100.

Yamaguti, S. 1939. Studies on the helminth fauna of Japan. Part 25. Tremtodes of Birds, IV. Japanese Journal of Zoology, Tokyo, 8: 129-210.

. 1971. Synopsis of digenetic trematodes of vertebrates. Tokyo, Keigaku Publishers, vol. 1, 1074p.

Recebido em 20.IV.2004; aceito em 18.X.2004.

Revista Brasileira de Zoologia 21 (4): 801-804, dezembro 2004 